\title{
Real-time and Power Hardware-in-the-loop Simulation of PEM Fuel Cell Stack System
}

\author{
Jee-Hoon Jung ${ }^{\dagger}$
}

\author{
${ }^{\dagger}$ Smart Grid Research Division, Korea Electrotechnology Research Institute, Changwon, Korea
}

\begin{abstract}
Polymer electrolyte membrane (PEM) fuel cell is one of the popular renewable energy sources and widely used in commercial medium power areas from portable electronic devices to electric vehicles. In addition, the increased integration of the PEM fuel cell with power electronics, dynamic loads, and control systems requires accurate electrical models and simulation methods to emulate their electrical behaviors. Advancement in parallel computation techniques, various real-time simulation tools, and smart power hardware have allowed the prototyping of novel apparatus to be investigated in a virtual system under a wide range of realistic conditions repeatedly, safely, and economically. This paper builds up advancements of optimized model constructions for a fuel cell stack system on a real-time simulator in the view points of improving dynamic model accuracy and boosting computation speed. In addition, several considerations for a power hardware-in-the-loop (PHIL) simulation are provided to clectrically emulate the PEM fuel cell stack system with power facilities. The effectiveness of the proposed PHIL simulation method developed on Opal RT's RT-Lab Matlab/Simulink based real-time engineering simulator and a programmable power supply is verified using experimental results of the proposed PHIL simulation system with a Ballard Nexa fuel cell stack.
\end{abstract}

Key Words: PEM fuel cell, Stack system, Power hardware-in-the-loop, Dynamic model, Real-time simulation

\section{INTRODUCTION}

Fuel cells provide a renewable and clean alternative solution to recent electric power generation technologies. The polymer electrolyte membrane (PEM) fuel cell has gained some acceptance in medium power commercial applications such as building backup power, grid tied distributed generation, and electric vehicles [1]. The PEM fuel cell's terminal conditions requires a power conditioning and electric regulation to interface the fuel cell power generator to its load or the grid [2], [3]. Load variations require a dynamic replenishment of the air and fuel to the fuel cell, while properly maintaining cell humidity and rejecting heat. Therefore, the development and testing of the fuel cell system in a laboratory environment would benefit from replacing fuel cells with accurate dynamic models and hardware simulators of the complete PEM fuel cell. This hardware emulator of the fuel cell system can provide useful testing and prototyping environments under various operating conditions without any worries about damages and malfunctions in practical fuel cell systems during their physical operations.

Since the spatial dimensions of a PEM fuel cell are complex, several internal quantities inside of the fuel cell are not measurable for outside users. However, proper mathematical models which describe electrochemical reactions inside of the

\footnotetext{
Manuscript received Nov. 15, 2010; revised Jan. 15, 2011

† Corresponding Author: jung.jeehoon@gmail.com

Tel: +82-55-510-1356, Mobile: +82-10-9811-3251

New \& Renewable Energy System Research Center, Smart Grid Research

Division, Korea Electrotechnology Research Institute (KERI), Korea
}

fuel cell can calculate the reaction results and electrical power generated from the reactions. One-dimensional models of thermal response and water management have been proposed for estimating the behavior of PEM fuel cell layers [4]. Dynamic fuel cell models predicting detailed internal performance using electrochemical reaction and thermal dynamic equations have been reported in [5], [6]. Model validations with commercial fuel cell systems are reported in [7], [8]. Computational fluid dynamic (CFD) simulations have also been used to corroborate the developed models [9].

However, the CFD simulation is not proper to a real-time simulation because of its high computational burden. Dynamic models based on algebraic computations have been developed for PEM fuel cell simulation [10], [11].Real-time simulation of fuel cell dynamic models has been used to improve fuel cell subsystems and dc-dc converters [12], [13]. Voltage and thermal dynamic models of PEM fuel cell layers were organized, and mathematical equations for computational software were proposed in [14]. Moreover, electrical and mathematical models of a Nexa fuel cell stack system, which is one of the well-known PEM fuel cell systems, have been developed to emulate its operation [15], [16].

In this paper real-time and power hardware-in-the-loop (PHIL) simulations are proposed to emulate a PEM fuel cell stack system in an electrical manner. This paper is an extended work of references [17], [18] including a PHIL simulation system with dynamics of subsystems and electrochemical effects. Fig. 1 shows a physical configuration of the PHIL simulation system of the PEM fuel cell stack using a real-time 
Fuel Cell Stack Power HIL

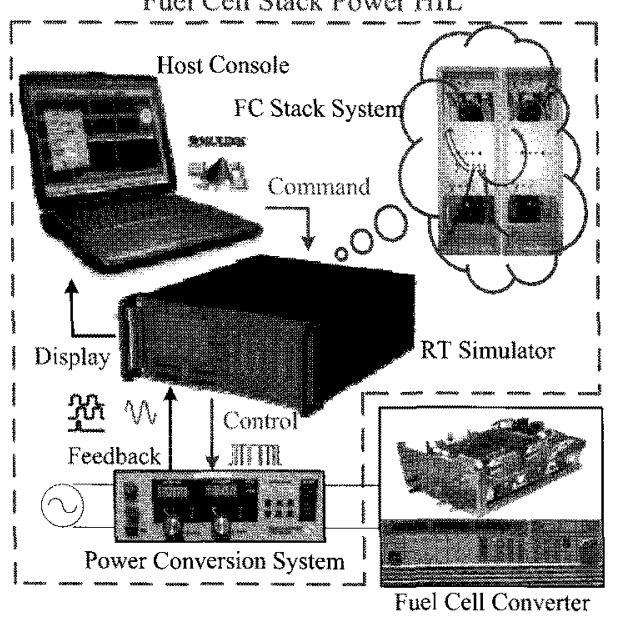

Fig. 1. Conceptual schematic of fuel cell power HIL simulation.

simulator. Fuel cell stack systems are expensive. In addition, a hydrogen supply system and other auxiliary systems require additional engineering and safety precautions for laboratory testing. The proposed real-time simulation-based PHIL can provide a fast, safe, and inexpensive way to accurately design and evaluate power converters and interfaces for fuel cell applications as well as other electrical system applications [19]-[21]. The mathematical representation of the fuel cell electrochemistry was extracted from [13], [14]. Mathematical equations of the terminal voltage, layer temperatures, oxygen excess ratio (OER), and fuel consumption are programmed as model blocks in MATLAB Simulink. The static and dynamic validations of the proposed simulation model compared to a Ballard Nexa fuel cell stack system are achieved using statistical inference methods. In addition, the proposed PHIL simulation system is implemented using the RT-LAB realtime engineering simulator and PQA III programmable power supply. Model considerations for a parallel computation and an interface between the real-time simulator and power hardware are discussed using experimental results of the PHIL simulation system.

\section{Dynamic Model of PEM Fuel Cell Stack}

In this section, the dynamic model of a PEM fuel cell which is composed of a terminal voltage model and a thermal layer model will be summarized using electrochemical and thermal dynamics theory. Detail derivation and description of these models are already introduced in references [14], [18]. It is noted that definitions of all variables and parameters used in equations of the terminal voltage model and the thermal layer model are already described in reference [18]. In addition, a fuel cell stack system and additional subsystems will be modeled using electrical and dynamic equations. Finally, effects of hydrogen and oxygen flow rate, and oxygen excess ratio will be considered to emulate the fuel cell stack system practically.

\section{A. Terminal Voltage and Thermal Layer Models}

Fuel cells provide a voltage dependent on operating conditions such as temperature, electrical load, and fuel and oxidant flow rates. The maximum possible cell potential, $V_{c}$, is the net output voltage given by (1) with the reversible cell potential, $V_{r v}$, and the irreversible potential, $V_{i r v}$ [14].

$$
V_{c}(i)=V_{r v}-V_{i r v} .
$$

The reversible cell potential, $V_{r v}$, can be modeled as the Nernst voltage. To calculate the Nernst voltage as a reversible cell potential, the saturation pressure of water at temperature $T$ $\left({ }^{\circ} \mathrm{C}\right), p_{\text {sat }}(T)\left(\mathrm{kgf} / \mathrm{cm}^{2}\right)$, and the partial pressures of hydrogen $p p_{\mathrm{H}_{2}}$ and oxygen $p p_{\mathrm{O}_{2}}$ are used as follows [14]:

$$
\begin{aligned}
E_{N}= & 1.229-8.15 \times 10^{-4}(T-298.15) \\
& +4.308 \times 10^{-5} T \cdot \ln \left(p p_{\mathrm{H}_{2}} \cdot \sqrt{p p_{\mathrm{O}_{2}}}\right) .
\end{aligned}
$$

The irreversible voltage loss is composed of the activation overpotential $V_{a c t}$, ohmic overpotential $V_{o h m}$, and concentration overpotential $V_{c o n}$.

$$
\begin{gathered}
V_{i r v}=-V_{a c t}+V_{o h m}+V_{c o n} \\
V_{a c t}=\frac{R T_{c a}}{\alpha_{c} F}\left[\frac{\alpha_{c}}{\alpha_{a}+\alpha_{c}} \ln \left(\frac{\gamma}{i_{h r} M_{H_{2} g}}\right)-\ln \left(\frac{-\gamma}{i_{o r} M_{O_{2} g}}\right)\right] \\
V_{o h m}=i\left[\sum_{j} R_{c, j}+\sum_{k} \frac{L_{c, k}}{\sigma_{c, k} A_{c, k}}+\int_{0}^{t_{p}} \frac{d z}{\sigma_{m}(\Delta \lambda(z))}\right] \\
V_{c o n}=\frac{R T_{c o}}{n_{e} F}\left(1+\frac{1}{\alpha_{m}}\right) \ln \left(\frac{\bar{i}_{L}}{\bar{i}_{L}-i}\right), i<\bar{i}_{L}
\end{gathered}
$$

where $\gamma=i\left[a_{120}(1-S) P_{t g}\right]^{-1}, \sigma_{m}$ is the conductivity profile of the membrane, $\Delta \lambda$ is the variation of the water content, and $\bar{i}_{L}$ is the average limiting current density, respectively.

The heat generation and a exchange can be described by proper governing equations [14], [18]. All parameters used in the equations are obtained from references and data sheets of a fuel cell power module made by Ballard Power Systems, which is the target PEM fuel cell system. The governing equations of a coolant channel which considers convection and radiation effects and a catalyst layer which reflects the heat generation from the electrochemical reaction as follows:

$$
\begin{gathered}
m_{l 1} \frac{d T_{l 1}}{d t}=Q_{c v}+Q_{r a d}+Q_{l 2} \\
m_{l 5} \frac{d T_{l 5}}{d t}=Q_{l 4}+Q_{l 6}+Q_{i n t, l 5}+Q_{r e s, l 5}
\end{gathered}
$$

where $Q_{c v}$ is the heat flow by forced and natural convection, $Q_{\text {rad }}$ is the heat flow by radiation, and $Q_{i n t, l 5}$ is the heat generation due to the electrochemical reaction and voltage overpotential, respectively.

\section{B. Considerations for Stack System}

In this subsection the single cell simulation model proposed in the previous subsections is extended to the fuel cell stack system under considerations of fuel cell stacking, operating temperature of the stack system, air compressor as a subsystem, and its power consumption. Fig. 2 shows a proposed simulation model structure of the fuel cell stack system which is composed of a cell model, stack model, and stack-cell interface. The cell model calculates terminal voltage and layer temperature of the single fuel cell. The stack model computes stack current and air mass flow of the 


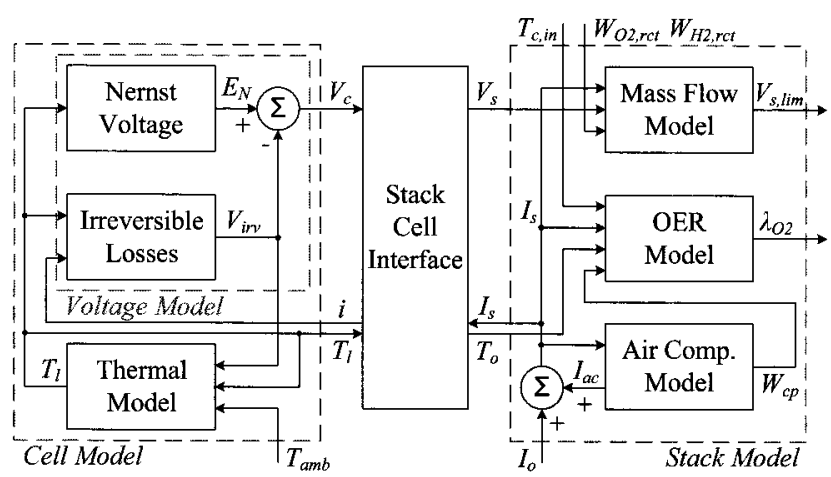

Fig. 2. Proposed simulation model structure of fuel cell system.

air compressor. In addition, power limitations influenced by oxygen and hydrogen reaction rates, and oxygen excess ratio of the fuel cell stack are simulated, which will be discussed in the next subsections. Cell and stack values of voltage, current, and temperature are transformed to each other by the stack-cell interface.

Total stack current $I_{s}$, composed of the stack output current $I_{o}$ and the subsystem current consumption $I_{s s}$, can be calculated using the cell current density $i$ and the effective area of cell $A_{\text {cell }}$ as in (9).

$$
I_{s}=I_{o}+I_{s s}=i A_{c e l l} \text {. }
$$

Stack voltage $V_{s}$ can also be calculated using the cell potential, $V_{c}$, and the number of cells, $N_{c e l l}$, under consideration of total stack resistance, $R_{s}$, and forward voltage drop of the stack diode, $V_{f}$, as in (10).

$$
V_{s}=V_{c} N_{c e l l}-R_{s} I_{s}-V_{f}
$$

In the Nexa fuel cell system, the dynamics of the output air mass flow of the air compressor can be modeled as follows [8]:

$$
W_{c p}(s)=\frac{0.1437 s^{2}+2.217 s+8.544}{s^{3}+3.45 s^{2}+7.324 s+5.745} V_{c r}
$$

where $V_{c r}$ is the air compressor's input voltage controlled by a Nexa control board, which can be expressed as in (12) using a curve fitting method from experimental data.

$$
V_{c r}=1.73 \times 10^{-3} I_{s}^{3}-0.128 I_{s}^{2}+3.62 I_{s}+0.352 .
$$

The current consumption of the air compressor, $I_{a c}$, can be modeled with respect to the air mass flow of the compressor as in (13).

$$
I_{a c}=-2.43 \times 10^{-5} W_{c p}^{3}+4.22 \times 10^{-3} W_{c p}^{2}-0.174 W_{c p}+3.83 \text {. }
$$

Power consumptions from other fuel cell subsystems such as a control board and cooling fan are included in $I_{a c}$ since they are smaller than the power consumption of the air compressor $\left(I_{s s} \approx I_{a c}\right)$.

\section{Effects of Hydrogen and Oxygen Reaction Rates}

The output power generated by the PEM fuel cell system is limited by the amount of supplied air (oxygen) and fuel (hydrogen). From the definition of current, $I=n F(d N / d t)$, the oxygen and hydrogen reaction rates (mass flows) are as follows:

$$
\begin{aligned}
W_{\mathrm{O}_{2}, r c t} & =M_{\mathrm{O}_{2}} \frac{N_{c e l l} I_{s}}{n_{\mathrm{O}_{2}} F} \\
W_{H_{2}, r c t} & =M_{H_{2}} \frac{N_{c e l l} I_{s}}{n_{\mathrm{H}_{2}} F}
\end{aligned}
$$

where $n_{\mathrm{O}_{2}}$ and $n_{H_{2}}$ is the mol number of electrons per mol of $\mathrm{O}_{2}$ and $\mathrm{H}_{2}$, respectively. From (14) and (15), the total stack current, $I_{s}$, generated from the fuel cell stack is given by (16) considering the consumption of specific hydrogen and oxygen mass flows; where $M_{\mathrm{O}_{2}}$ and $M_{H_{2}}$ is the molecule mass number of $\mathrm{O}_{2}$ and $\mathrm{H}_{2}$, respectively.

$$
I_{s}=\frac{n_{\mathrm{O}_{2}} F}{N_{\text {cell }} M_{\mathrm{O}_{2}}} W_{\mathrm{O}_{2}, r c t}=\frac{n_{\mathrm{H}_{2}} F}{N_{\text {cell }} M_{H_{2}}} W_{H_{2}, r c t} \text {. }
$$

Therefore, the maximum stack current, $I_{s, \max }$, is limited by (17).

$$
I_{s, \max }=\frac{F}{N_{\text {cell }}} \cdot \min \left[\frac{n_{\mathrm{O}_{2}}}{M_{\mathrm{O}_{2}}} W_{O_{2}, r c t}, \frac{n_{H_{2}}}{M_{H_{2}}} W_{H_{2}, r c t}\right] \text {. }
$$

Since the maximum stack power, $P_{s, \max }$, is limited by $I_{s, \max }$, the stack output voltage can be decreased by the limited amount of oxygen and/or hydrogen supplied from the air compressor and fuel tank. This can be expressed as follows:

$$
\begin{gathered}
P_{s, \max }=N_{c e l l} V_{c}\left(i_{\max }\right) I_{s, \max } \\
V_{s, \lim }\left(I_{s}\right)=\frac{P_{s, \max }}{I_{s}}=N_{c e l l} V_{c}(i) \frac{I_{s, \max }}{I_{s}}
\end{gathered}
$$

where $i_{\max }$ is the maximum cell current density under limited air and/or fuel supplies. From (19), oxygen or hydrogen starvation implies a decrease in cell and stack voltage. This phenomenon is a serious danger to the fuel cell system because it can induce hot spots or burn-through on the surface of the membrane layer.

\section{Oxygen Excess Ratio (OER)}

To prevent oxygen starvation in the membrane, the air compressor controller in the fuel cell system regulates the OER in the cathode by controlling the amount of oxygen mass flow from the air compressor to the inlet of the cathode [8]. The oxygen excess ratio is defined by (20).

$$
\lambda_{O_{2}}=\frac{W_{O_{2}, i n}}{W_{O_{2}, r c t}} .
$$

The oxygen reaction rate, $W_{\mathrm{O}_{2}, \text { rct }}$, is obtained from (14) and the mass flow of $\mathrm{O}_{2}$ through the inlet of the cathode, $W_{\mathrm{O}_{2}, \text { in }}$, can be calculated as follows:

$$
W_{\mathrm{O}_{2}, i n}=\gamma_{\mathrm{O}_{2}, r c t} W_{a i r, i n}
$$

where $W_{\text {air,in }}$ is the dry air flow in the cathode inlet and $\gamma_{\mathrm{O}_{2}, \text { rct }}$ is the molar mass relation between oxygen and dry air given by (22); where $M_{a i r, i n}$ is the molar mass of the dry air at the cathode inlet, $M_{O_{2} g}$ and $M_{N_{2} g}$ is the mol fraction of $\mathrm{O}_{2}$ and $\mathrm{N}_{2}$ in the gas phase, respectively.

$$
\gamma_{O_{2}, r c t}=\frac{M_{O_{2} g} M_{O_{2}}}{M_{a i r, i n}}
$$




$$
M_{a i r, i n}=M_{O_{2} g} M_{O_{2}}+M_{N_{2} g} M_{N_{2}} .
$$

Equation (23) assumes that other gases in the air are negligible.

In addition, $W_{a i r, i n}$ can be calculated from (24); where $\omega_{i n}$ is the humidity ratio which is the relation between the masses of water vapor and dry air in the cathode inlet air, $M_{v}$ is the water vapor molar mass, $\phi_{c, i n}$ is the inlet air humidity, $p_{c, \text { in }}$ is the cathode inlet pressure, $p_{v, \text { in }}$ and $p_{d, i n}$ are the partial pressures of water vapor and dry air, respectively.

$$
\begin{gathered}
W_{a i r, i n}=\frac{1}{1+\omega_{i n}} W_{i n} \\
\omega_{i n}=\frac{M_{v}}{M_{a i r, i n}} \cdot \frac{p_{v, i n}\left(T_{c, i n}\right)}{p_{d, i n}\left(T_{c, i n}\right)} \\
p_{c, i n}=1.0033+\left(2.1 \times 10^{-3}\right) W_{c p}-\left(475.7 \times 10^{-6}\right) I_{s} \\
p_{v, i n}(T)=\phi_{c, i n} p_{s a t}(T) \\
p_{d, i n}(T)=p_{c, i n}-p_{v, i n}(T) .
\end{gathered}
$$

It is noted that (26) comes from an empirical testing reported in [8]. The cathode inlet air flow, $W_{\text {in }}$, can be calculated from (29); where $M_{a m}$ is the inlet air molar mass.

$$
\begin{gathered}
W_{i n}=\frac{W_{c p}}{22.4 \times 60} M_{a m} \\
M_{a m}=\frac{p_{d, i n}\left(T_{o}\right)}{p_{c, i n}} M_{a i r, i n}+\frac{p_{v, \text { in }}\left(T_{o}\right)}{p_{c, i n}} M_{H_{2} O} .
\end{gathered}
$$

\section{REAL-TIME SIMULATION FOR PHIL}

The terminal voltage, stack interface, and thermal dynamic models are implemented using Matlab/Simulink. The Simulink models are compiled using the Opal-RT's RT-Lab real-time simulator. The platform provides parallel computing hardware capability, and accompanying transient solvers and component libraries. Optimal model construction for real-time simulation needs to take the simulator hardware architecture into consideration. This section introduces the model development of the fuel cell stack system on the real-time simulator as well as the parallel computation approaches using more than two processing cores to reduce model computation time.

\section{A. Model Structure of Real-time Simulation}

The electrochemical equations of the fuel cell stack system presented in the previous section show the strong correlations among cell voltage, output current, layer temperatures, and pressure inside of the cell. The pressure and current are determined by air and fuel supply as well as loading conditions. Operating temperature depends on the thermal dynamics and heat generations of the fuel cell layers as well as the cooling subsystem of the fuel cell stack. Also, layer temperature is influenced by the irreversible voltage loss and cell current. These interdependencies are shown in Fig. 3 which illustrates a real-time simulation model of the fuel cell stack system. In Fig. 3 , the dynamic system blocks consist of a thermal dynamic block, cell voltage block, and stack interface block. Additional OpComm and OpMonitor blocks are needed by RT-LAB for its real-time simulation. The OpComm block creates a realtime communication link between the model and simulator's

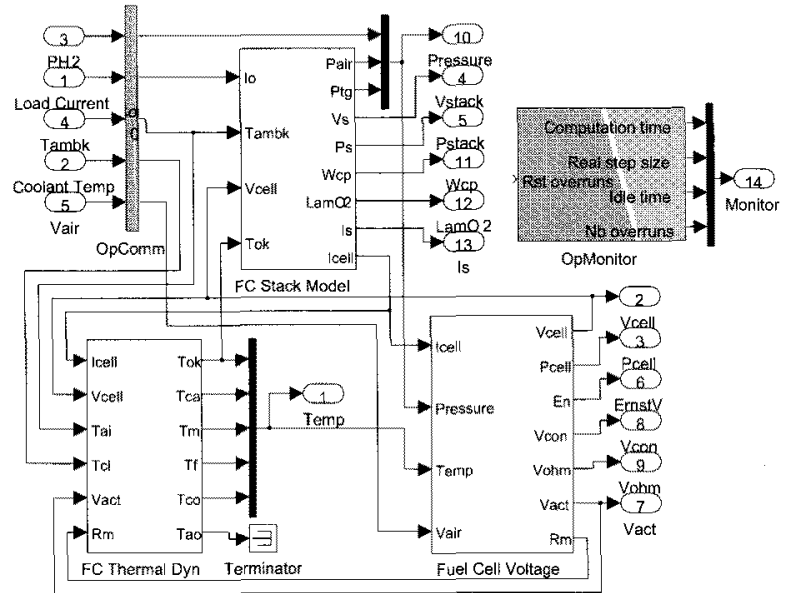

Fig. 3. Dynamic model of the PEM fuel cell stack system using MATLAB Simulink.

input/output. The OpMonitor block adds real-time monitoring capability to the simulation.

The fuel cell voltage model is composed of four voltage blocks: Nernst voltage block, activation loss block, ohmic loss block, and concentration loss block. Input cell current and output cell voltage have range limitations because the cell voltage model provides meaningful results only under proper operating voltage and current ranges. The thermal dynamic model is composed of thermal dynamic blocks of cell layers and an air temperature block. The air temperature block calculates the temperature of the output air. In the cell layer block, temperatures of adjacent layers become inputs of each block to calculate thermal exchanges between each adjacent layer. The stack interface model calculates mass flow rate of hydrogen, OER, pressures, air mass flow of the air compressor, and total stack current. In addition, the stack interface model transforms the cell voltage to the stack voltage and the stack current to the cell current.

\section{B. Elimination of Algebraic Loops in the Model}

Algebraic loops are present in the simulation model of the fuel cell stack system that contains dynamics of its subsystems. Output states are directly dependent on previous outputs due to feedback-based control loops. Simulation blocks have input ports with direct feedthrough; it means that the output of these simulation blocks cannot be computed without knowing the values of the signals entering the blocks at these input ports. This forces the simulator to solve each simulation time step iteratively, therefore it makes the simulation slowing down. This algebraic loop generally occurs when an input port with direct feedthrough is driven by the output of the same simulation block. Moreover, in the real-time simulation, the algebraic loop can occur among simulation blocks computed in parallel. An simple concept of the algebraic loop is in Fig. 4. Mathematically, this loop implies that the output of the simulation block is an algebraic state $z$ constrained to equal the first input $x$ plus $y$ minus $z$ (i.e., $z=x+y-z$ ). The solution of this mathematical loop is $z=(x+y) / 2$, but most algebraic loops cannot be solved by inspection because the simulation is based on a logical computation. 


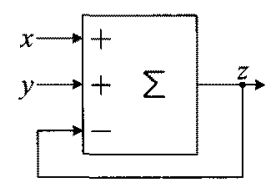

Fig. 4. Simple example of the algebraic loop.

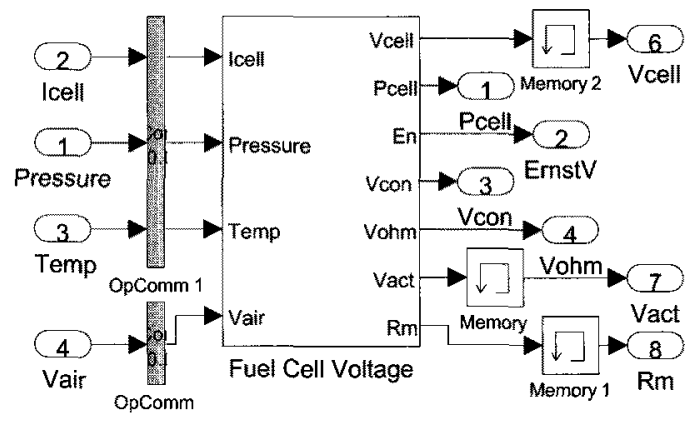

Fig. 5. Terminal voltage model with memory blocks.

Memory blocks or low-pass filters can be placed in the algebraic loop in order to rectify this problem. Since the realtime simulation is configured for fixed-time step simulation, memory blocks are used to break the algebraic loop. Fig. 5 shows the terminal voltage model of the PEM fuel cell stack system with memory blocks. The feedback signals of the cell potential, activation loss, and membrane resistance generated from the terminal voltage model block are connected to the thermal dynamic model and the stack interface model as input signals in Fig. 3. Therefore, three memory blocks are used to break algebraic loops created between model blocks computed in parallel. This memory block produces more deterministic behavior than the low-pass filter in a fixedtime step environment.

\section{Model Considerations for Parallel Computation}

The real-time simulation environment has limitations of simulator performance and computation time. The real-time simulation machine has fixed and limited processing power. The computation time is a more critical constraint because all computations should be completed within a fixed simulation time step. If not, overruns will cause errors to occur in the simulation, and they can propagate to the entire process. Model computation speed can be improved if one makes use of the various processor cores. Simulation models should be separated so they only exchange priority signals which are state or state-derived between computation subsystems. By separating the model into one master and two slave subsystem blocks, the RT-LAB real-time simulator can assign each model to different cores for parallel computing.

In the real-time simulation model, the master and slave subsystems have to compute and send their outputs before they read their inputs within the same simulation step for optimizing parallel executions. In addition, the state or state-derived signals have to be identified to enable parallel computation of subsystems. Fig. 6 shows the recommended structure for the parallel execution using three processing cores. A state can be defined as an output computed only from preceding inputs or outputs; output blocks of the subsystems have to

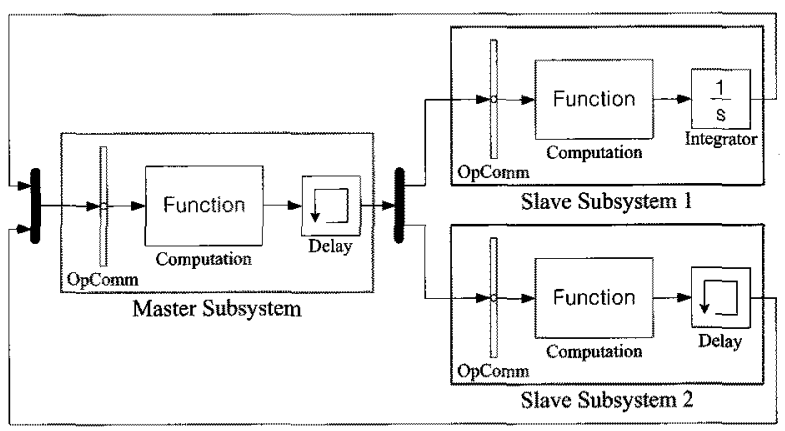

Fig. 6. Model structure for parallel execution using three processing cores.

be delayed. The terminal voltage and stack interface model blocks require memory blocks in their outputs to give an unit delay. The output state of the thermal dynamic model is, however, naturally connected to an integrator block, so it does not need any blocks for delaying the signal. This parallel computation is based on multi-core simulators; however, multicore processing technology is widely used in general purpose microprocessors. Consequently, the proposed considerations for the parallel computation are general algorithms and can be widely applied to any commercial multi-core real-time simulators.

It should also be noted that the calculation time is highly dependent on the degree of model separation and distribution. If the computation burden of the simulation model is equally divided into several models that are in turn assigned to multiple cores, the total computation time is expected to be approximately divided by the number of cores used compared to the single core case. However, if the model is not equally divided, the computation time will follow the calculation speed of the most complex one of the distributed models. Thus, it is safe to say that the minimum number of cores needed for a distributed real-time simulation is that which allows the complete system dynamics to be simulated with no loss in accuracy when the model is separated approximately equally.

\section{Simulation AND EXPERIMENTAL RESULTS}

In this section performance and validity of the proposed fuel cell dynamic model are discussed. Static model characteristics such as stack voltage and power of the proposed model are illustrated with respect to the stack current and operating temperature including their validation test. Dynamic model characteristics such as the stack current, stack voltage, hydrogen flow rate, OER, and coolant temperature are also shown. In addition, their model accuracies are verified using experimental data of the Ballard Nexa fuel cell stack system. The improvement of the computation speed in the real-time simulator is tested using three cores. Finally, experimental results of the proposed PHIL simulation system are presented with a Magna-Power's PQA III programmable power supply.

\section{A. Static Model Validation}

Validation of the proposed fuel cell stack model was done using experimental data with a $1.2 \mathrm{~kW}$ Nexa Ballard fuel cell stack system. Fig. 7 shows the output voltage characteristics of the stack terminal voltage model according to the load 


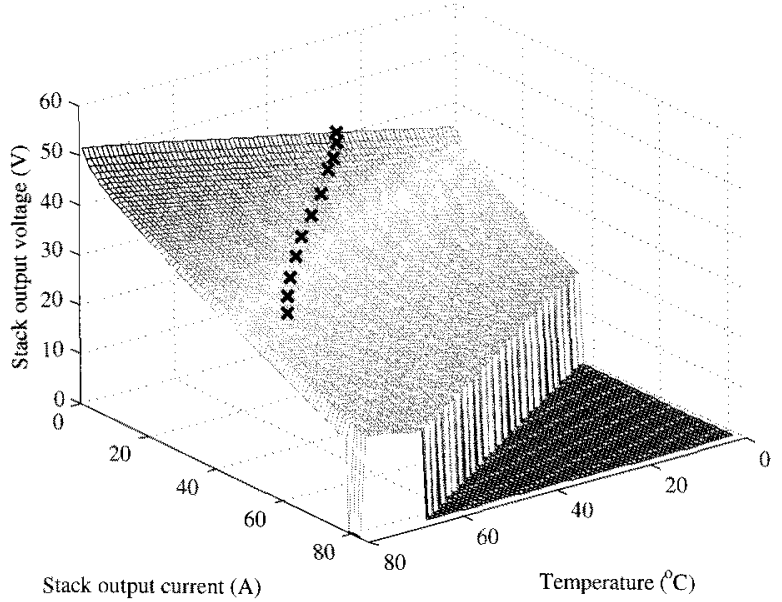

Fig. 7. Output voltage characteristics of the fuel cell stack terminal voltage model with experimental data.

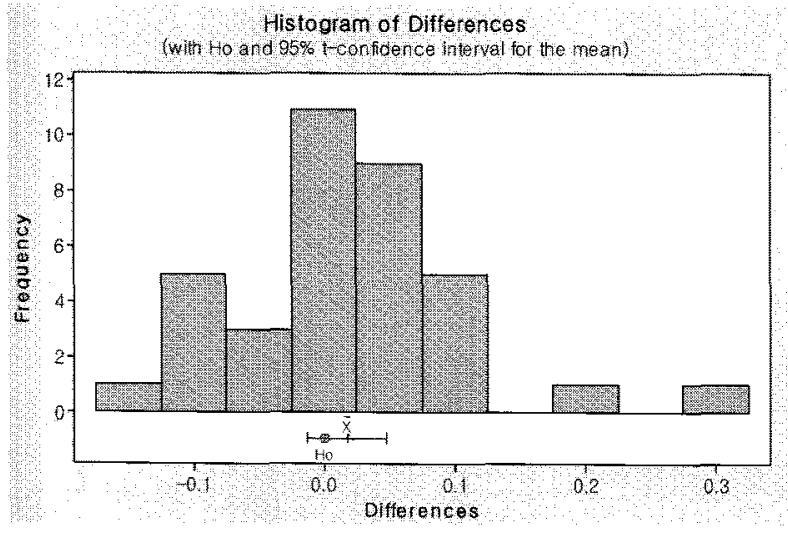

Fig. 8. Paired t-test result of the stack voltage model.

current and operating temperature. In Fig. 7, the experimental data (cross) are laid on the three dimensional surface of the simulated voltage curve. In addition to the visual inspection of the data, a mathematical approach was also used. The paired t-test procedure was used to compute the mean difference between two populations [22]. It was carried out using the statistical software MINITAB. Fig. 8 shows the paired t-test result of the static response of the stack terminal voltage illustrated in Fig. 7. Fig. 8 means that the difference between the two data sets can be ignored with a $95 \%$ confidence level.

\section{B. Dynamic Model Validation}

Fig. 9 shows the comparison between the experimental data and the simulation model response in the stack current, stack voltage, hydrogen flow rate, and OER. The good agreement in transient dynamics and steady state values between the simulation model and experimental results hold for all the fuel cell's performance factors. Fig. 10 shows the comparison of thermal dynamic data with respect to loads of $10 \mathrm{~A}$ and $30 \mathrm{~A}$ evolving over 20 minutes. The ambient temperature in this case was $27.5^{\circ} \mathrm{C}$, which was also used as the initial temperature in the simulation. In Fig. 10 the proposed thermal dynamic model of the fuel cell layers calculates the experimental temperature data for various load conditions accurately.

For a detailed validation of the model accuracy, another error analysis has been carried out using the mean relative

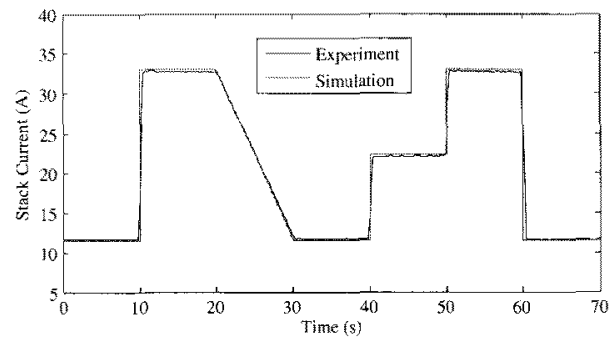

(a)

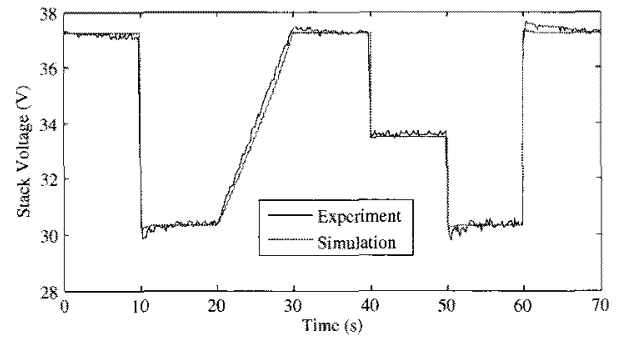

(b)

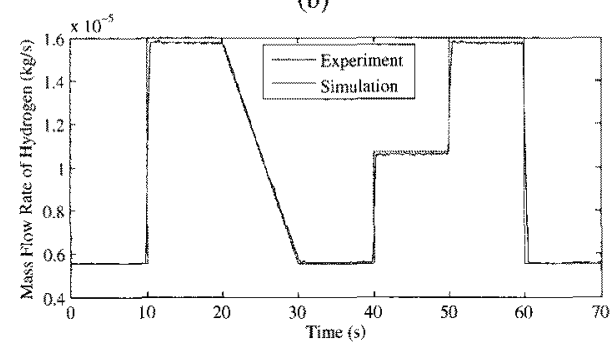

(c)

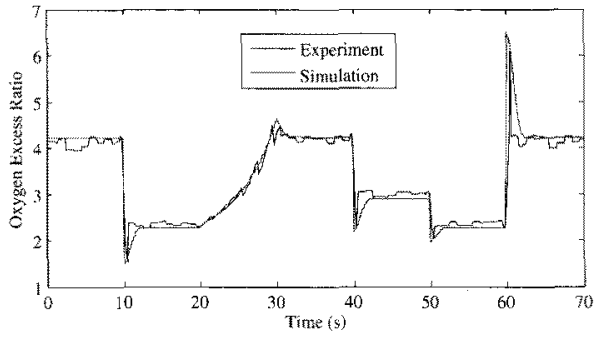

(d)

Fig. 9. Comparison between experimental data and simulation model response (a) Stack current, (b) Stack voltage, (c) Mass flow rate of $\mathrm{H}_{2}$, (d) Oxygen excess ratio.

error (MRE) criterion [8] as follows:

$$
\operatorname{MRE}(\%)=100 \times \frac{1}{N} \sum_{i=1}^{N}\left|\frac{D_{i}^{e}-D_{i}^{s}}{D_{i}^{e}}\right|
$$

where $D_{i}^{e}$ and $D_{i}^{s}$ represent the experimental and simulated data sets, and $N$ is the number of samples, respectively. Using (31), the MRE criterion is applied to the dynamic responses of the stack voltage, the mass flow rate of $\mathrm{H}_{2}$, the OER, and the thermal dynamic responses. As a result, the relative mean errors of the first three parameters in the same order are $0.73 \%, 0.09 \%$, and $2.31 \%$, respectively. In addition, the errors of thermal dynamics at $10 \mathrm{~A}$ and $30 \mathrm{~A}$ load conditions are calculated as $3.83 \%$ and $6.56 \%$, respectively.

\section{Improved Computation Speed}

Fig. 11 (a) shows the real-time simulation computation time of the base fuel cell model. Fig. 11 (b), (c), and (d) show 


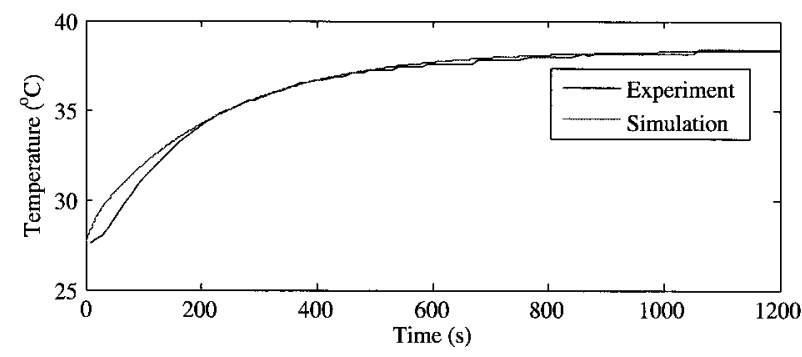

(a)

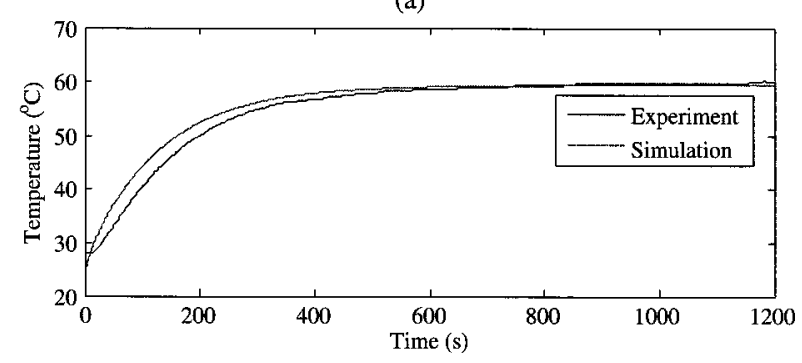

(b)

Fig. 10. Comparison of thermal dynamic data with respect to load conditions: (a) 10 A load, (b) 30 A load.

TABLE I

COMPUTATION TIME OF THE DISTRIBUTED MODELS

\begin{tabular}{c|ccc}
\hline Model & Single Core & Dual Cores & Triple Cores \\
\hline Single & $36 \mu \mathrm{s}$ & - & - \\
M.Volt & - & $6.9 \mu \mathrm{s}$ & $5.5 \mu \mathrm{s}$ \\
S.Temp & - & $0.8 \mu \mathrm{s}$ & $1.2 \mu \mathrm{s}$ \\
S.Int & - & - & $4.2 \mu \mathrm{s}$ \\
\hline
\end{tabular}

the computation times of the master voltage model, the slave temperature model, and the slave interface model using the proposed distribution method model with three computing cores, respectively. Table I shows the improvement of the computation speed of the proposed distribution model comparing with the base model and a distribution model with two cores. The computations of the base model were completed in an average of $36 \mu \mathrm{s}$. Using the parallel computation technique with two cores, the time consumptions of the terminal voltage model including the stack interface and the thermal dynamic layer model is $6.9 \mu \mathrm{s}$ and $0.8 \mu \mathrm{s}$, respectively. Finally, the computation time of the three distributed models; the voltage, temperature, and interface models using three cores is $5.5 \mu \mathrm{s}$, $1.2 \mu \mathrm{s}$, and $4.2 \mu \mathrm{s}$, respectively.

From the result, it is verified that the parallel computation technique can improve the computation speed in the real-time simulation. In addition, the more process cores can reduce the more computation time. Consequently, the improvement in computation time brought about by the proposed model development method using three cores is on the order of $85 \%$. In addition, this computation speed is $20 \%$ more faster than the simulation using two cores. However, the computation speed is not linearly proportional to the number of cores since the entire simulation model is not equally separated into the distributed models. In addition, model complexities are increased in the model separation for multi-cores.

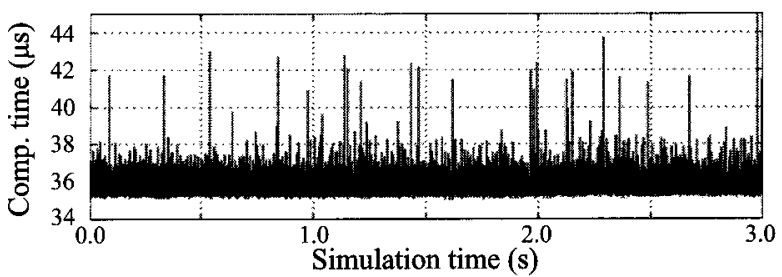

(a)

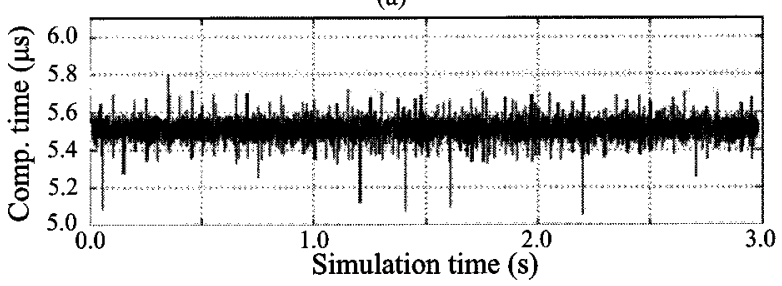

(b)

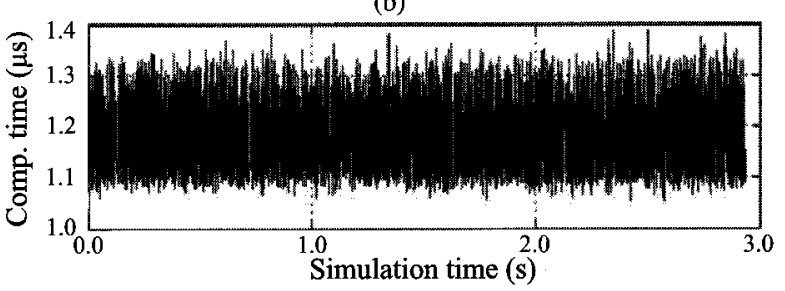

(c)

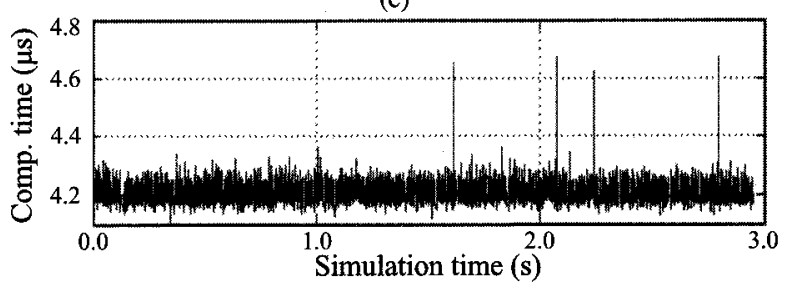

(d)

Fig. 11. Computational time consumptions of the RT simulation models: (a) Base model, (b) Master voltage model, (c) Slave temperature model, (d) Slave interface model.

\section{PHIL Simulation Results}

Fig. 12 (a) shows the experimental setup of the proposed PHIL simulation system for emulating the PEM fuel cell stack. Fig. 12 (b) shows the block diagram of its interfacing configurations. In Fig. 12 (b), there are two computer interfaces: console PC and monitoring PC is connected to the realtime simulator and the electronic load for monitoring variables, commanding executions, and generating load patterns, respectively. A real power interface is only used between the programmable power supply and the electronic load, which is a physical emulator as a power source and a power application of the fuel cell system, respectively. An ethernet interfaces is used for fast communication between the console PC and the real-time simulator. All other interfaces are designed by serial communication lines to transfer and receive digital data such as measurements, status, and commands.

Fig. 13 shows experimental waveforms of the load current and stack voltage in the PHIL simulation. The load current pattern is the same as the pattern in the real-time simulation. According to the load current variation, the stack voltage simulated by the real-time simulator is generated through the programmable power supply. Between the real-time simulator and the programmable power supply, the reference voltage and 


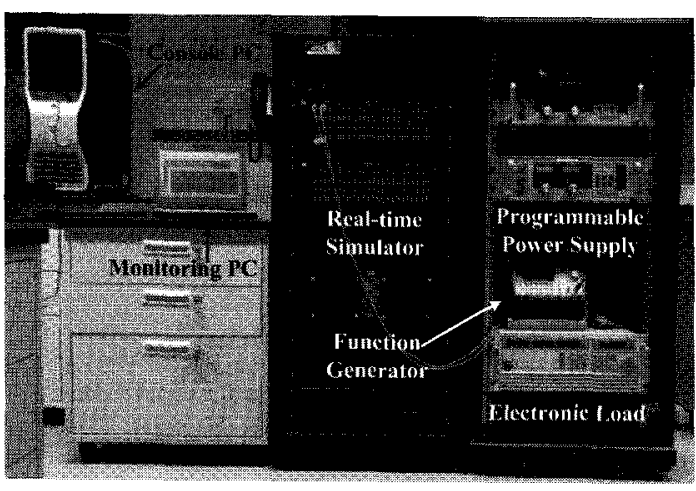

(a)

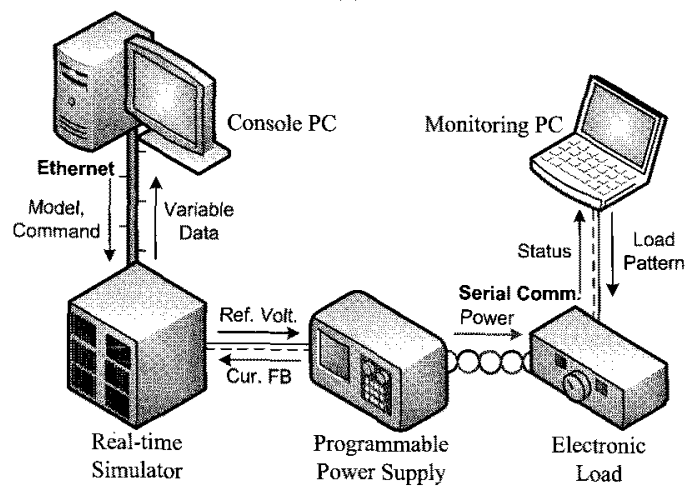

(b)

Fig. 12. Proposed PHIL simulation system: (a) Photograph of the experimental setup, (b) Block diagram of the PHIL simulation system.

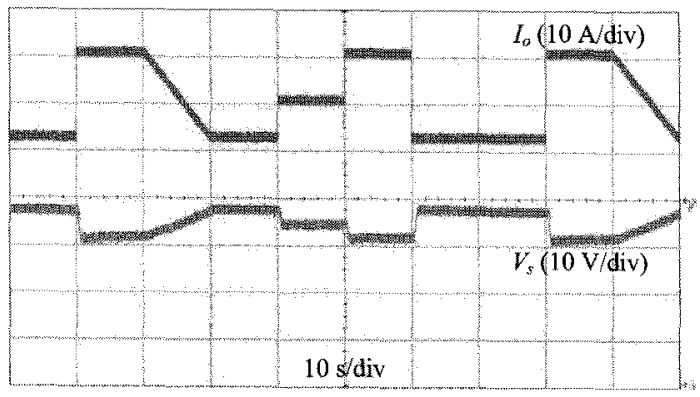

Fig. 13. Experimental load current and stack voltage waveforms of the PHIL simulation.

the current feedback signals are transferred and received using the RS232 serial communication with 19200 bit per second. Depending on the communication speed, additional delays can affect the dynamics of the emulated system using the PHIL simulation.

\section{CONCLUSIONS}

A distributed real-time simulation model and a PHIL simulation method of a PEM fuel cell stack system for emulating its electrical dynamics has been presented. The proposed simulation model represents the electrical voltage and thermal dynamic behavior of the fuel cell stack system considering several effects such as electrical loss in the stack system, dynamics of the subsystems, reaction rates of hydrogen and oxygen, and the OER. For the PHIL simulation, the simulation model structure is optimized and considers the elimination of the algebraic loop for the real-time simulation. Using multiple computing cores, the parallel computation is also adopted to improve the computation speed in the real-time simulation. The proposed simulation model was tested and verified using the RT-LAB real-time simulator and the Ballard Nexa fuel cell system. Consequently, it have been confirmed that the model is well matched to the target fuel cell system using the statistical verification tools with $85 \%$ improvement in computation speed over the base model. In addition, the PHIL simulation was achieved using power hardware such as the programmable power supply and the patterned electronic load. The proposed model accurately and quickly calculated system dynamics in real time. As a result, the proposed PHIL simulation method using the real-time simulation model properly emulates electrical behaviors of the target PEM fuel cell stack system.

\section{ACKNOWLEDGMENT}

This work was supported by a National Priorities Research Program (NPRP) grant from the Qatar National Research Fund (QNRF), and the National Research Foundation of Korea Grant funded by the Korean Government [NRF-2009-352D00109].

\section{REFERENCES}

[1] B. D. Solomon and A. Banerjee, "A global survey of hydrogen energy research. development and policy," Energy Policy, Vol. 34, No. 7, pp. 781-792, May 2006.

[2] W.-C. Lee, S.-J. Jang, S.-S. Kim, S.-W. Lee, and C.-Y. Won, "A fuel cell generation system with a new active clamp sepic-flyback converter," Journal of Power Electronics, Vol. 9, No. 1, pp. 26-35, Jan. 2009.

[3] L. Zhang, X. Yang, W. Chen, and X. Yao, "An isolated soft-switching bidirectional buck-boost inverter for fuel cell applications," Journal of Power Electronics, Vol. 10, No. 3, pp. 235-244, May 2010.

[4] A. Rowe and X. Li, "Mathematical modeling of proton exchange membrane fuel cells," Joumal of Power Sources, Vol. 102, No. 1-2, pp. 82-96, Dec. 2001

[5] S. Pasricha and S. R. Shaw, "A dynamic PEM fuel cell model," IEEE Trans. Energy Convers., Vol. 21, No. 2, pp. 484 - 490, Jun. 2006.

[6] A. J. D. Real, A. Arce and C. Bordons, "Development and experimental validation of a PEM fuel cell dynamic model," Jouma of Power Sources, Vol. 173, No. 1, pp. 310-324, Nov. 2007.

[7] M. V. Moreira and G. E. D. Silva, "A practical model for evaluating the performance of proton exchange membrane fuel cells," Renewable Energy, Vol, 34, No. 7, pp. 1734-1741, Jul. 2009.

[8] C. Ramos-Paja, R. Giral, L. Martinez-Salamero, J. Romano, A. Romero, and G. Spagnuolo, "A PEM fuel cell model featuring oxygen excess ratio estimation and power electronics interaction," IEEE Trans, Ind. Eletron., Vol. 57, No. 6, pp. 1914-1924, Jun. 2010.

[9] C. Siegel, "Review of computational heat and mass transfer modeling in polymer-electrolyte-membrane (PEM) fuel cells," Energy, Vol, 33, No. 9, pp. 1331-1352, Sep. 2008.

[10] J.-G. Lim and S.-K. Chung, "Implementation of a fuel Cell dynamic simulator," Journal of Power Electronics, Vol. 7, No. 4, pp. 336-342, Oct. 2007.

[11] X. Kong and A. M. Khambadkone, "Modeling of a PEM Fuel-Cell Stack for Dynamic and Steady-State Operation Using ANN-Based Submodels," IEEE Trans. Ind. Electron., Vol. 56, No. 12, pp. 49034914, Dec. 2009.

[12] M. Meiler, O. Schmid, M. Schudy, and E. P. Hofer, "Dynamic fuel cell stack model for real-time simulation based on system identification;" Journal of Power Sources, Vol. 176, No. 2, pp. 523-528, Feb. 2008.

[13] S.-Y. Choe, J.-W. Ahn, J.-G. Lee, and S.-H. Baek, "Dynamic simulator for a PEM fuel cell system with a PWM DC/DC converter," IEEE Trans. Energy Convers., Vol. 23, No. 2, pp. 669-680, Jun. 2008.

[14] C. Spiegel, PEM fuel cell modeling and simulation using Matlab, Academic Press/Elsevier, San Diego, 2008.

[15] F. Gao, B. Blunier, A. Miraoui, and A. El-Moudni, "Cell layer level generalized dynamic modeling of a PEMFC stack using VHDL-AMS language," Int. J. Hydrogen Energy, Vol. 34, No. 13, pp. 5498-5521, Jul. 2009. 
[16] H.-I. Kim, C. Y. Cho, J. H. Nam, D. Shin, and T.-Y. Chung, "A simple dynamic model for polymer electrolyte membrane fuel cell (PEMFC) power modules: Parameter estimation and model prediction," Int. J. Hydrogen Energy, Vol. 35, No. 8, pp. 3656-3663, Apr. 2010.

[17] J.-H. Jung, S. Ahmed, and P. Enjeti, "Fast computation methods of PEM fuel cell dynamic models for real-time simulation," IEEE International Power Electronics Conference (IPEC), pp. 954-961, 2010.

[18] J.-H. Jung and S. Ahmed, "Dynamic model of PEM fuel cell using realtime simulation techniques," Journal of Power Electronics, Vol. 10, No 6, pp. 739-748, Nov. 2010

[19] W. Ren, M. Steurer, and T. L. Baldwin, "An effective method for evaluating the accuracy of power hardware-in-the-loop simulations," IEEE Trans. Ind. Appl., Vol. 45, No. 4, pp. 1484-1490, Jul./Aug. 2009.

[20] M. Steurer, C. S. Edrington, M. Sloderbeck, W. Ren, and J. Langston, "A megawat1-scale power hardware-in-the-loop simulation setup for motor drives," IEEE Trans. Ind. Electron., Vol. 57, No. 4, pp. 1254-1260, Apr. 2010.

[21] A.-L. Allegre, A. Bouscayrol, J.-N. Verhille, P. Delarue, E. Chattot, and S. El-Fassi, "Reduced-scale-power hardware-in-the-loop simulation of an innovative subway," IEEE Trans. Ind. Electron., Vol. 57, No. 4, pp. 1175-1185, Apr. 2010.

[22] R. E. Walpole and R. H. Myers, Probability and Statistics for Engineers and Scientists 5th Edition, Prentice-Hall, New Jersey, chap. 10, 1993.

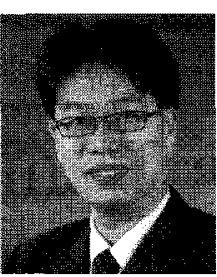

Jee-Hoon Jung was born in Suwon, Korea, in 1977. He received his B.S., M.S., and Ph.D. degrees from the Department of Electronic and Electrical Engineering. Pohang University of Science and Technology (POSTECH), Pohang, Korea, in 2000, 2002, and 2006, respectively. He was a Senior Research Engineer in the Digital Printing Division of Samsung Electronics Co., Ltd., Suwon, Korea from 2006 to 2009 . He was also a Postdoctoral Research Associate in the Electrical and Computer Engineering Department of Texas A\&M University at Qatar (TAMUQ), Doha, Qatar from 2009 to 2010 . He is currently a Senior Researcher in New \& Renewable Energy System Research Center, Smart Grid Research Division of Korea Electrotechnology Research Institute (KERI), Changwon, Korea. His research interests include dc-dc converters, switched mode power supplies, motor drives and diagnosis systems, digital control and signal processing algorithms, digitally controlled power electronics, and power conversions for renewable energy. Recently, he is researching on realtime and power hardware-in-the-loop (PHIL) simulations of the renewable energy sources. Dr. Jung is a member of the IEEE Industrial Electronics Society, IEEE Power Electronics Society, and the Korean Institute of Power Electronics (KIPE) 